\title{
Efeito de farinhas espessantes na aceitabilidade do fishburguer de aruanã branco (Osteoglossum bicirrhosum)
}

\author{
Effect of thickening flours on the acceptability of white arowana \\ (Osteoglossum bicirrhosum) fish burgers
}

\author{
Hérlon Mota ATAYDE'; Ézia Lima de AMORIM²; Elicelma Santana de BELEZA ${ }^{3}$; \\ Euclides Luís QUEIROZ-DE-VASCONCELOS ${ }^{4}$; Maria José Mendonça de OLIVEIRA ${ }^{5}$
}

${ }^{1}$ Autor para correspondência, engenheiro de pesca, Doutor em Ciências Pesqueiras nos Trópicos, Docente da Universidade
Federal do Oeste do Pará, Rua Vera Paz, s/n, Unidade Bloco Modular Tapajós, bairro Salé, CEP 68040-255, Santarém - PA,
herlon.atayde@ufopa.edu.br
${ }^{2}$ Tecnóloga em Alimentos pela Universidade do Estado do Amazonas, gideaomartins090@gmail.com
${ }^{3}$ Tecnóloga em Alimentos pela Universidade do Estado do Amazonas, senhor.meupastor2017@gmail.com
${ }^{4}$ Engenheiro de pesca, Mestrando em Ciência Animal e Recursos Pesqueiros pela Universidade Federal do Amazonas,
euclides_luis15@hotmail.com
${ }^{5}$ Engenheira de pesca, Mestre em Ciências Pesqueiras nos Trópicos pela Universidade Federal do Amazonas,
mary_olliver@hotmail.com Recebido em: 30-07-2020; Aceito em: 25-05-2021

\section{Resumo}

Considerando a crescente demanda de produtos com elevado valor nutricional e de rápido e fácil preparo, mais o atendimento de consumidores sensíveis ao glúten, essa pesquisa teve como objetivo elaborar e analisar sensorialmente o fishburguer de aruanã branco com diferentes farinhas espessantes, além de avaliar o rendimento do filé. Para a obtenção das formulações, os filés foram triturados e misturados aos demais ingredientes até obtenção de uma massa homogênea. Esta foi repartida em três porções iguais, cada uma acrescida de diferentes farinhas (arroz, milho ou trigo) para espessamento (constituindo três formulações). Porções de $80 \mathrm{~g}$ de cada formulação foram moldadas em formato circular, entre sacos plásticos, obtendo-se os fishburgueres, que foram submetidos ao congelamento para posterior utilização. Para a análise sensorial, os fishburgueres foram fritos em óleo quente $\left(180^{\circ} \mathrm{C}\right)$ por cerca de 2 minutos de cada lado e servidos ainda mornos para os julgadores que, no teste de aceitação aplicado (escala hedônica de 5 pontos), avaliaram os seguintes atributos: cor, odor, sabor e textura. Nessa análise, foram utilizados 70 julgadores não treinados, habituados ao consumo de pescado. $O$ rendimento de filé foi de $35,7 \pm 2 \%$. Não houve diferença estatisticamente significativa quanto à aceitação dos atributos e formulações, obtendo médias próximas a 4,0 (gostei), independentemente dos agentes espessantes. A aceitabilidade $(72,85 \%)$ do fishburguer de aruanã utilizando a farinha de milho como agente espessante demonstrou que essa é uma alternativa atrativa e capaz de favorecer o consumo desse produto por indivíduos sensíveis ao glúten, além de agregar valor e ampliar o mercado consumidor dessa espécie.

Palavras-chave adicionais: análise sensorial; hambúrguer de peixe; milho.

\begin{abstract}
Considering the growing demand for products with high nutritional value and that are easy-to-eat, plus serving gluten-sensitive consumers, this research aimed to develop and to sensory analyze fish burgers made from white arowana using different thickening flours, and to evaluate the yield of the fish fillet. To obtain the formulations, the fillets were smashed and mixed to other ingredients until it turned into a homogenous paste. This paste was separated into three equal portions, each one added with different flours (rice, maize, and wheat) for thickening (constituting three formulations). Portions weighing $80 \mathrm{~g}$ of each formulation were formatted in a circular shape, between plastic bags, obtaining the fish burgers, submitted to freezing until the next step. For sensory test, the fish burgers were fried in hot oil $\left(180^{\circ} \mathrm{C}\right)$ for about two minutes per side and offered still warm to tasters that, in the acceptance test applied (five points hedonic scale), evaluated the following attributes (color, odor, flavor, and texture). In this sensory test, 70 untrained tasters were heard, all were accustomed to eating fish foods. The fillet yield was $35.7 \pm 2 \%$. There was no significant difference regarding the tasters' preference for each attribute and formulations, with medium index next to 4.0 (I liked), regardless of the thickening flour. The acceptability $(72.85 \%)$ of white arowana fish burger formulation with maize flour as thickening agent is an attractive alternative to add economic value and extend the fish consumption market, also favoring the consumption by gluten-intolerant people.
\end{abstract}

Additional keywords: corn; fish hamburger; sensory analyses. 


\section{Introdução}

O pescado é uma importante fonte natural de minerais (macro e micro), todos indispensáveis para muitos processos vitais, como o funcionamento adequado do sistema cardiovascular, contratilidade muscular, condutividade neural, metabolismo celular e outras diversas atividades fisiológicas (Balami et al., 2019; Tacon et al., 2020).

Exceto para a Região Norte do país, o Brasil apresenta um dos mais baixos índices de consumo de pescado, e esse quadro é, parcialmente, uma consequência do desconhecimento dos brasileiros quanto à riqueza nutricional desse produto na alimentação, de fatores culturais e renda (Viana et al., 2016).

Apesar do peixe apresentar características nutricionais suficientes para estimular o seu consumo, há muitos fatores interferentes (por exemplo, espécies com muitas espinhas intramusculares, custo elevado, dificuldade de preparação, baixa variabilidade de derivados) capazes de serem contornados por meio do desenvolvimento ou aprimoramento de produtos de fácil preparo (Lopes et al., 2016).

Entre esses produtos de fácil preparo, o hambúrguer de peixe ou fishburguer é definido como um aglomerado de carne de peixe triturado ou moído, no qual são adicionados vários ingredientes que promovem modificações e melhorias na cor, textura, suculência e percepção bucal (Boscolo \& Feiden, 2007).

Em alimentos, os agentes espessantes podem conferir funcionalidade e agregar valor nutritivo ao produto. Por funcionalidade, compreende-se como a propriedade atribuída a determinado alimento que, quando consumido regularmente, promove efeitos benéficos adicionais à saúde do consumidor, além da nutrição básica (Granato et al., 2020). Como exemplo, cita-se a menor incidência de câncer cólon-retal em consumidores mais frequentes de alimentos ricos em fibras alimentares (Ocvirk et al., 2019), como aquelas presentes no milho e derivados.

Entre os espessantes do fishburguer, alternativas à farinha de trigo precisam ser testadas porque, entre os potenciais consumidores, há aqueles portadores de sensibilidade ao glúten, seja por doença celíaca ou não. Conforme Caio et al. (2019), a doença celíaca é uma condição autoimune caracterizada por um perfil sorológico e histológico desencadeado pela ingestão de glúten em indivíduos geneticamente predisponentes, capazes de ocasionar diversos sintomas, como exemplo os clássicos diarreia, má absorção intestinal e perda de peso, ainda os não-clássicos constipação, anemia, osteoporose, desordens neurológicas, dermatites, entre outras. Os portadores dessa doença correspondem a 0,5-1,0\% da população mundial, com maior prevalência nos países ocidentais.

O aruanã branco (Osteoglossum bicirrhosum) é um peixe pertencente à família Osteoglossidae, popularmente conhecido como aruanã, sulamba, macacod'água, baiano, arawana. Atinge mais de $1 \mathrm{~m}$ de comprimento e pode alcançar $5 \mathrm{~kg}$ de peso total. É normalmente comercializado in natura no mercado da Região
Norte do Brasil e, fora desse mercado, como filé congelado (Santos et al., 2006).

Diante da baixa disponibilidade comercial de produtos que atendam os requisitos de rápido e fácil preparo, com alto valor nutritivo e livre de espinhas, pesquisas que apresentem inovações para o consumo de peixes em geral ganham importância.

Nesse artigo, foi verificado o efeito sensorial de diferentes agentes espessantes na aceitabilidade do fishburguer de aruanã, buscando apontar fórmulas diferenciadas e funcionais para esse produto, capazes de atender os consumidores sensíveis ao glúten e a praticidade requerida pelo homem moderno.

\section{Material e métodos}

Coleta, biometria e rendimento em filé sem pele e sem espinhas de aruanã branco

Exemplares de aruanã branco $(n=10)$ foram adquiridos nas feiras da cidade de Tapauá, na região do Rio Purus, no Estado do Amazonas. Esse município é $565 \mathrm{~km}$ distante da capital amazonense e acessível somente por via aérea ou fluvial. Para verificação do rendimento em filé sem pele, conforme Souza \& Inhamuns (2011), foram efetuadas medições de comprimento padrão (em centímetros) e peso (em quilogramas) do peixe inteiro e do filé após retirada das espinhas. Os dados obtidos foram utilizados para cálculo de rendimento (em percentagem) do filé sem pele e sem espinhas.

\section{Obtenção do triturado lavado de aruanã branco}

Os exemplares de aruanã in natura foram lavados com água clorada, em seguida descamados. 0 peixe descamado foi novamente submetido à lavagem com água clorada, em seguido foi filetado, retirando-se pele e espinhas intramusculares. Os filés sem pele e espinhas foram lavados com água clorada, drenados e triturados em moinho elétrico. O triturado obtido foi submetido por duas vezes ao seguinte processo, conforme recomendado por Neiva \& Gonçalves (2011): foi adicionado em uma mistura de água com gelo $\left(10^{\circ} \mathrm{C}\right)$ e bicarbonato de sódio (para pH 7), durante 10 minutos, na proporção de 3:1 (água: peixe). Ao final desse processo, foi efetuada nova drenagem no triturado, após inserção dele em um saco de pano previamente higienizado e submetido à prensagem manual. A carne triturada e lavada foi embalada em sacos plásticos (no máximo $2 \mathrm{~kg}$ ) ligeiramente achatados e em seguida foi congelada, para posterior utilização no preparo dos fishburgueres.

\section{Preparo do fishburguer de aruanã branco}

A carne triturada e lavada do peixe foi misturada com vários ingredientes em comum, mas variando-se os agentes espessantes, sendo então obtidas três formulações (Tabela 1). A formulação controle foi baseada no protocolo de aulas práticas do Laboratório de Tecnologia do Pescado da Universidade Federal do Amazonas, modificando-se somente o tipo 
de tempero industrializado. As farinhas utilizadas como espessante, objeto desse estudo, foram trigo (tipo especial e sem fermento, para formulação controle), milho (tipo flocada) e arroz (tipo integral).

Tabela 1 - Formulações dos fishburgueres de aruanã branco (Osteoglossum bicirrhosum). Formulations of white arowana (Osteoglossum bicirrhosum) fish burgers.

\begin{tabular}{lccc}
\hline \multirow{2}{*}{$\begin{array}{l}\text { Descrição dos ingredientes e seus percentuais em } \\
\text { cada formulação }\end{array}$} & \multicolumn{3}{c}{ Formulações } \\
\cline { 2 - 4 } & ${ }^{*}$ F1 (trigo) & F2 (milho) & F3 (arroz) \\
\hline Triturado lavado de aruanã branco (74,6\%) & 1000,0 & 1000,0 & 1000,0 \\
Gordura vegetal hidrogenada (3,0\%) & 40,0 & 40,0 & 40,0 \\
Cebola (Allium cepa, 2,7\%) & 36,7 & 36,7 & 36,7 \\
Alho (Allium sativum, 0,9\%) & 11,7 & 11,7 & 11,7 \\
${ }^{* *}$ Tempero verde (2,1\%) & 28,3 & 28,3 & 28,3 \\
${ }^{* * *}$ Tempero industrializado (1,1\%) & 15,0 & 15,0 & 15,0 \\
Sal de cozinha (0,5\%) & 6,7 & 6,7 & 6,7 \\
Pimenta-do-reino (Piper nigrum, 0,1\%) & 1,7 & 1,7 & 1,7 \\
Farinha de trigo (Triticum sp., 14,9\%) & 200,0 & - & - \\
Farinha de milho (Zea mays, 14,9\%) & - & 200,0 & - \\
Farinha de arroz (Oryza sativa, 14,9\%) & - & - & 200,0 \\
\hline
\end{tabular}

${ }^{*}$ Formulação controle; ${ }^{* *}$ Mistura de coentro (Coriandrum sativum), cebolinha (Allium schoenprasum) e chicória (Cichorium intybus var. intybus), encontrada em feiras populares, convertidas em gramas (1:1:1); ${ }^{* \star \star}$ Tipo Sazón $\Theta$, com coentro e toque de pimenta. ${ }^{*}$ Control formulation; ${ }^{* *}$ Mixture of coriander (Coriandrum sativum), chive (Allium schoenprasum) and chicory (Cichorium intybus var. intybus), found in popular markets, converted in grams (1:1:1); ${ }^{* *}$ Sazón® label, with coriander and touch of pepper.

Os ingredientes (exceto a farinha) foram homogeneizados em liquidificador e misturados com o triturado de aruanã para a obtenção de uma massa uniforme, que foi dividida em três porções iguais, cada uma adicionada dos espessantes. Para obtenção dos fishburgueres, essas misturas foram divididas em porções de $80 \mathrm{~g}$ cada, as quais foram moldadas em formato circular, entre sacos plásticos, e submetidas ao congelamento.

\section{Análise sensorial dos fishburgueres de aruanã branco}

A análise sensorial foi efetuada por 70 julgadores não-treinados, de ambos os sexos (não quantificados), habituados ao consumo de pescado, com idades entre 13 e 52 anos. Os menores de idade (42,86\%) estavam acompanhados do responsável no momento da degustação.

A avaliação de aceitação por meio de escala hedônica apresentava pontuação de 1 a 5 (sendo as afirmações para cada ponto equivalentes a: 1 = desgostei muito, 2 = desgostei, 3 = não gostei/nem desgostei, 4 = gostei e 5 = gostei muito) (Minim, 2013). Optou-se por utilizar essa escala com menor gradação devido sua maior facilidade de compreensão pelos julgadores, pois foi uma atividade inédita para todos.

As amostras foram codificadas, o que tornou a associação entre código e fórmula de conhecimento sigiloso do preparador do teste sensorial. Para cada formulação, foi solicitado que cada julgador atribuísse uma pontuação para os atributos cor e odor antes da inserção da amostra na boca, e para sabor e textura, após inserção na boca. A pontuação atribuída foi registrada em ficha individual.
Nessa pesquisa, optou-se pelo termo odor porque as literaturas técnico-científicas [por exemplo, Dutcosky (2019) e Raul et al. (2018)] apontam que este termo se refere à percepção ortonasal (ocorrida pela aspiração, antes da introdução do alimento na boca ato solicitado aos julgadores), no lugar de aroma, retronasal (ocorre após a introdução do alimento na boca), apesar da normativa brasileira (Associação Brasileira de Normas Técnicas, 2017) indicar que, no Brasil, aroma refere-se ao odor de alimentos.

Para degustação, os fishburgueres congelados foram previamente fritos em óleo quente (aproximadamente $180^{\circ} \mathrm{C}$ ) por cerca de 2 minutos de cada lado e separados por formulação. Foram servidos mornos (máx. 2 min após fritura), simultaneamente, em pratos descartáveis brancos acompanhados de água mineral e bolacha salgada, para limpeza do paladar entre as amostras. Uma porção de cada formulação (aprox. $20 \mathrm{~g}$ ) foi alocada em um campo delimitado do prato, contendo a respectiva codificação. As degustações ocorreram em ambiente aberto, porém coberto, sendo as mesas dispostas de forma que os julgadores não poderiam efetuar qualquer comunicação, verbal ou gestual. Ao final, para cada julgador foi atribuída uma pontuação média por formulação, obtendo-se assim a análise global do produto, e consequentemente, a indicação da formulação com maior aceitação.

Adicionalmente, foi calculado o índice de aceitabilidade, somando-se a quantidade de pontos positivos (gostei e gostei muito) atribuídos para cada formulação. Foi considerado aceitável o fishburguer com índice $\geq 70 \%$, conforme Bispo et al. (2004). 


\section{Análise estatística}

Os dados obtidos foram submetidos a análises descritivas e inferenciais. A homogeneidade dos dados foi atestada pelo método de Levene, sendo a nãonormalidade dos dados atestada pelo método de Anderson-Darling. Devido a essa última condição, utilizou-se o teste não-paramétrico Kruskal-Wallis para verificação de similaridades ou diferenças entre as formulações de fishburgueres. Todos os testes foram analisados ao nível de significância de $5 \%$, por meio do programa estatístico Minitab 19.1.

\section{Resultados e discussão}

Os dados biométricos obtidos para a aruanã (O. bicirrhosum) estão presentes na Tabela 2 .

Tabela 2 - Biometria e rendimento em filé (sem pele e espinhas) de aruanã branco (Osteoglossum bicirrhosum). Biometry and yield of white arowana (Osteoglossum bicirrhosum) fillets (without skin and bones).

\begin{tabular}{ccccc}
\hline $\begin{array}{c}\text { Aruanã } \\
\text { (número de exemplares) }\end{array}$ & $\begin{array}{c}\text { Comprimento } \\
\text { padrão* } \\
(\mathrm{cm})\end{array}$ & $\begin{array}{c}\text { Peso total* } \\
(\mathrm{kg})\end{array}$ & $\begin{array}{c}\text { Peso do filé sem } \\
\text { pele e espinhas* } \\
(\mathrm{kg})\end{array}$ & $\begin{array}{c}\text { Rendimento* } \\
(\%)\end{array}$ \\
\hline 10 & $63,25 \pm 3,24$ & $2,10 \pm 0,32$ & $0,750 \pm 0,13$ & $35,7 \pm 2,0$ \\
\hline
\end{tabular}

*Os valores são apresentados no formato média \pm desvio padrão. * Values are presented in mean \pm standard deviation.

Com base no comprimento, pode-se perceber que todos os exemplares utilizados já ultrapassavam aquele relacionado à primeira maturação sexual $(51 \mathrm{e}$ $56 \mathrm{~cm}$; fêmeas e machos, respectivamente), conforme a pesquisa de Cortegano et al. (2014). Portanto, entre os exemplares comercializados na cidade de TapauáAM, predominam aqueles que atendem ao tamanho mínimo de captura preconizado para a Bacia Amazônica (Amazonas, 2001).

Pode-se observar que o rendimento do filé de aruanã, mesmo com a retirada das espinhas intramusculares, foi superior ao apresentado por Costa (2006) $29,15 \pm 1,48 \%$ - para essa mesma espécie. Essa variação no rendimento de filé é observada em outras espécies de peixes e depende da experiência do manipulador, da idade e estado nutricional dos exemplares, entre outros fatores (Ørnholt-Johansson et al., 2017).

As médias obtidas na análise sensorial apontaram todas as formulações com tendência para "gostei" na aceitação global (Tabela 3).

Tabela 3 - Valores médios da análise sensorial de três formulações de fishburguer elaborados de carne triturada de aruanã (Osteoglossum bicirrhosum) com diferentes agentes espessantes. Averages of the sensory analysis of three fish burgers formulations made from minced flesh of white arowana (Osteoglossum bicirrhosum) with different thickening agents.

\begin{tabular}{|c|c|c|c|c|c|}
\hline \multirow{2}{*}{$\begin{array}{l}\text { Formulação do } \\
\text { fishburguer }\end{array}$} & \multicolumn{4}{|c|}{${ }^{*}$ Atributos sensoriais } & \multirow{2}{*}{$\begin{array}{c}\text { Análise global do } \\
\text { produto* }\end{array}$} \\
\hline & Cor & Odor & Sabor & Textura & \\
\hline${ }^{* *} \mathrm{~F} 1$ (trigo) & $3,8 \pm 0,9^{a}$ & $3,6 \pm 1,0^{a}$ & $3,9 \pm 1,0^{a}$ & $3,8 \pm 1,0^{a}$ & $3,8 \pm 0,8^{a}$ \\
\hline F2 (milho) & $3,8 \pm 1,0^{a}$ & $3,7 \pm 1,0^{a}$ & $3,6 \pm 1,0^{a}$ & $3,5 \pm 1,1^{a}$ & $3,7 \pm 0,8^{a}$ \\
\hline F3 (arroz) & 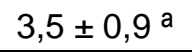 & $3,9 \pm 1,0^{a}$ & $3,5 \pm 1,2^{a}$ & $3,5 \pm 1,0^{a}$ & $3,6 \pm 0,8^{a}$ \\
\hline
\end{tabular}

* Dados apresentados em média \pm desvio-padrão, ${ }^{* *}$ formulação controle. ${ }^{*}$ Data provided in average \pm standard deviation, ${ }^{* *}$ control formulation.

Nota: As letras "a" sobrescritas na mesma coluna representam inexistência de diferenças estatisticamente significativas entre as formulações ao nível de $5 \%$ de significância. The superscript letters "a" in the same column represent no statistical differences between formulations at the $5 \%$ level of significance.

Também não se detectou diferença estatisticamente significativa quanto à aceitação dos julgadores em relação a qualquer um dos atributos, todos obtendo média com tendência para "gostei", independente dos agentes espessantes.

Por comparação direta entre as formulaçõesteste com a controle, mas analisando individualmente cada atributo (Figura 1), percebeu-se que para a cor, aquela contendo milho foi similar, mas a farinha de arroz imprimiu perdas nesse produto. Em contraponto, no odor, a formulação arroz imprimiu mais agrado aos julgadores. Para os demais atributos - sabor e textura, ambas as formulações em teste obtiveram demérito.

Quanto à aceitação global, Silva \& Fernandes
(2010), ao desenvolverem formulação de fishburguer de corvina (Argyrosomus regius), observaram $85 \%$ de aceitabilidade e nota média de 8,6 (tendência para a afirmação "gostei muitíssimo" em escala hedônica de 9 pontos). Por sua vez, Oliveira (2004) elaborou fishburguer de acará-prata (Chaetobranchus semifasciatus) com adição de proteína de soja e obteve média de 8,0 (tendência para a afirmação "gostei muito" em escala hedônica de 9 pontos). Ambas as pesquisas diferem do presente estudo quanto à aceitação global, pois todas alcançaram tendência para a maior escala. Essa constatação aponta a necessidade de melhorias nas dosagens dos ingredientes utilizados, assim como a verificação de novos itens a serem adicionados na mistura. 


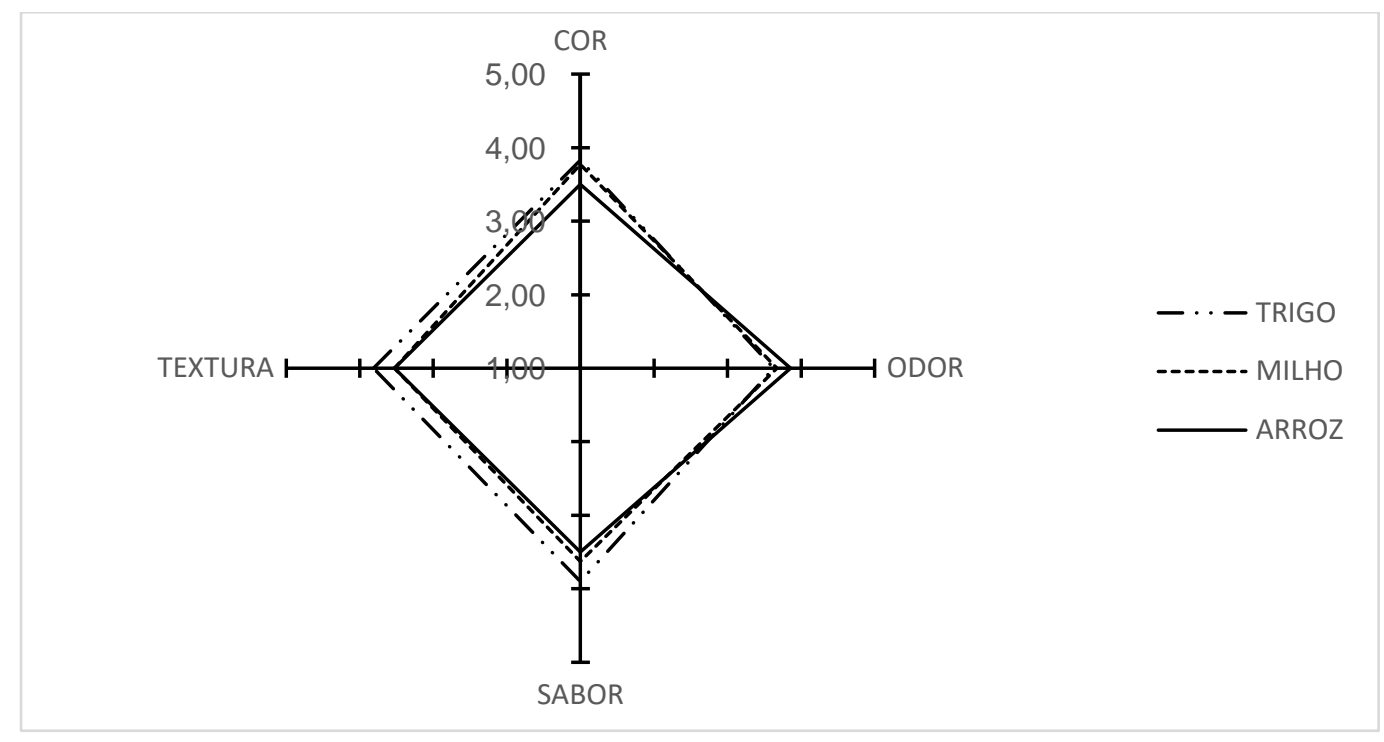

Figura 1. Representação tipo radar para a análise sensorial de formulações de fishburgueres elaborados com carne triturada de aruanã branco (Osteoglossum bicirrhosum) utilizando diferentes agentes espessantes. Radar representation for the sensory analysis of fish burger formulations made with white arowana meat (Osteoglossum bicirrhosum) using different thickening agents.

Na pesquisa de Cedola et al. (2017), foi obtida a média de aceitação global igual a 7,5 (escala hedônica de 9 pontos) para o fishburguer de atum (espécie não determinada) picado elaborado com farinha de batata - o espessante em sua formulação controle - em detrimento daquele utilizando a farinha de bagaço de azeitona. Apesar de sua pesquisa não apresentar as afirmações para cada ponto da escala sensorial, presume-se que o consumo dessa formulação controle foi "muito prazerosa", demonstrando que a farinha de batata é um importante espessante.

O fishburguer de armau (Pterodoras granulosus) picado elaborado por Munhoz \& Campozano (2018) utilizou como principais espessantes o amido e o creme de cebola, em formulação única. Pela análise sensorial (escala hedônica de 9 pontos), obteve o índice 8,07 na aceitação global, equivalente a afirmação "gostei muito".

Raúl et al. (2018) verificaram a aceitabilidade de fishburguer de biquara (Haemulon plumierii) contendo os espessantes farinha de trigo (em diferentes concentrações - 0, 1, 2 e 3\% - em cada formulação) e amido de milho ( $1 \%$ nas quatro formulações). Percebeu-se aumento da aceitação conforme a concentração de farinha de trigo aumentava, fato atribuído à concomitante diminuição do cheiro de peixe do produto, alcançando o índice de 6,61 (tendência para a afirmação "gostei moderadamente"; escala hedônica de 9 pontos).

Essas pesquisas permitem constatar que o uso de outros tipos de farinhas na elaboração de fishburgueres tem importância, principalmente devido ao incremento sensorial obtido nessas iniciativas. Adicionalmente, apontam a viabilidade e aceitação de fishburguer obtidos das mais diferentes espécies de peixes.

Uma análise comparativa mais aprofundada dessas pesquisas com o presente estudo é dificultada pela diferença entre os ingredientes utilizados, além das características metodológicas empregadas nelas. Essa variação certamente é influenciada pelas características gastronômicas culturais e disponibilidade de itens em cada local.

A análise sensorial da presente pesquisa permitiu a verificação do índice de aceitabilidade por formulações, que obtiveram os seguintes resultados: miIho $(72,85 \%)$, arroz $(64,29 \%)$ e controle $(71,43 \%)$ (Tabela 4). Portanto, entre os espessantes não-convencionais aplicados ao fishburguer de aruanã, constatouse que somente o milho foi aceitável, atingindo índice superior ao controle. Considerando a ausência de glúten na farinha de milho, o uso desse ingrediente em fishburgueres merece ser considerado, pois beneficiaria os consumidores portadores de sensibilidade ao glúten, sem excluir os demais indivíduos.

\section{Conclusões}

O uso da farinha de milho como espessante do fishburguer de aruanã branco é uma alternativa atrativa para esse produto de fácil preparo porque, além de aumentar o consumo de peixes e não comprometer a sua aceitabilidade, estende o mercado consumidor para os indivíduos sensíveis ao glúten. Recomendamse pesquisas para verificação do incremento nutricional, funcional e da viabilidade econômica desse produto. 
Tabela 4 - Análise pormenorizada da classificação obtida pelas formulações de fishburguer de aruanã branco (Osteoglossum bicirrhossum) por cada ponto da escala sensorial. Detailed analysis of the classification obtained from the formulations of the white arowana (Osteoglossum bicirrhossum) fish burgers for each point of the sensory scale.

\begin{tabular}{|c|c|c|c|c|}
\hline \multirow{3}{*}{$\begin{array}{l}\text { Classificação do ponto } \\
\text { da escala sensorial }\end{array}$} & \multirow{3}{*}{ Ponto da escala sensorial } & \multicolumn{3}{|c|}{ Formulações } \\
\hline & & ${ }^{*} \mathrm{~F} 1$ (trigo) & F2 (milho) & F3 (arroz) \\
\hline & & \multicolumn{3}{|c|}{ **Índices obtidos } \\
\hline \multirow[t]{3}{*}{ Pontos de rejeição } & Desgostei muito & $0 / 0,00$ & $1 / 1,43$ & $0 / 0,00$ \\
\hline & Desgostei & $2 / 2,86$ & $3 / 4,29$ & $4 / 5,72$ \\
\hline & Subtotal & $2 / 2,86$ & $4 / 5,72$ & $4 / 5,72$ \\
\hline \multirow[t]{2}{*}{ Ponto de neutralidade } & Não desgostei, nem gostei & $18 / 25,72$ & $15 / 21,43$ & $21 / 30,00$ \\
\hline & Subtotal & $18 / 25,72$ & $15 / 21,43$ & $21 / 30,00$ \\
\hline \multirow[t]{3}{*}{ Pontos de aceitação } & Gostei & $32 / 45,71$ & $44 / 62,85$ & $36 / 51,43$ \\
\hline & Gostei muito & $18 / 25,72$ & $7 / 10,00$ & $9 / 12,86$ \\
\hline & Subtotal & $50 / 71,43$ & $51 / 72,85$ & 45 / 64,29 \\
\hline
\end{tabular}

* Formulação controle, ${ }^{* *}$ Em quantidade absoluta / relativa, em \%. * Control formulation, ${ }^{* *}$ Absolut / relative values in \%. Nota: Índices $\geq 70 \%$ na linha subtotal de "pontos de aceitação" significam que a formulação de fishburguer foi aceita pelos julgadores. Indexes $\geq 70 \%$ in subtotal line of "acceptation points" means that fish burger formulation was accepted by food tasters.

\section{Referências}

Amazonas (2001) Lei Ordinária $n^{\circ} 2.713$, de 28 de dezembro de 2001 - Dispõe sobre a política de proteção à fauna aquática e de desenvolvimento e aqüicultura sustentável no Estado do Amazonas. Disponível em: $<$ https://sapl.al.am.leg.br/norma/7168 > (Acesso em 10 nov 2019).

Associação Brasileira de Normas Técnicas (2017) ABNT NBR ISO 5492: Análise sensorial - vocabulário. ABNT.

Balami S, Sharma A, Karn R (2019) Significance of nutritional value of fish for human health. Malaysian Journal of Halal Research Journal 2(2). doi: 10.2478/mjhr-2019-0012

Bispo ES, Santana LRR, Carvalho RDS, Leite CC, Lima MAC (2004) Processamento, estabilidade e aceitabilidade de marinado de vongole (Anomalocardia brasiliana). Food Science and Technology 24(3):353-356. doi: 10.1590/S0101-20612004000300008

Boscolo WR, Feiden A (2007) Industrialização de tilápia. UNIOESTE. p.127.

Caio G, Volta U, Sapone A, Leffler DA, Giorgio RD, Catassi C, Fasano A (2019) Celiac disease: a comprehensive current review. BMC Medicine 17(1):142. doi: 10.1186/s12916-019-1380-z

Cedola A, Cardinali A, Nobile MA, Conte A (2017) Fish burger enriched by olive oil industrial by-product. Food Science \& Nutrition 5(4). doi: 10.1002/fsn3.461
Cortegano C, Pinedo L, Ibanez L, Ramírez M, Ruiz P (2016) Produtividade e características reprodutivas do aruanã, Osteoglossum bicirrhosum (Osteoglossiformes: Osteoglossidae), no Lago Grande, bacia do Rio Putumayo, Peru. Biota Amazônia 4(4):21-26. doi: 10.18561/2179-5746/biotaamazonia.v4n4p21-26

Costa TV (2006) Identificação de Novas espécies para criação em cativeiro: pescado capturado no estado do Amazonas. UFRRJ (Dissertação em Zootecnia).

Dutcosky SD (2019) Análise Sensorial de Alimentos. 5ed, Champagnat-PUCPress, 540p.

Granato D, Barba FJ, Kovačević, DB, Lorenzo JM, Cruz AG, Putnik P (2020) Functional foods: product development, technological trends, efficacy testing, and safety. Annual Review of Food Science and Technology 11: 93-118. doi: 10.1146/annurev-food032519-051708

Lopes IG, Oliveira RG, Ramos FM (2016) Perfil do consumo de peixes pela população brasileira. Biota Amazonia 6(2): 62-65. doi: 10.18561/2179-5746/biotaamazonia.v6n2p62-65

Minim VPR (2013) Análise Sensorial: estudos com consumidores. 2ed, UFV, 332p.

Munhoz CL, Campozano RJ (2018) Elaboração de fishburguer do peixe armau (Pterodoras granulosus). Revista Inova Ciência \& Tecnologia 4(1): 20-24.

Neiva CRP, Gonçalves AA (2011) Carne mecanicamente separada de pescado e surimi. In: Gonçalves AA (org.) Tecnologia do Pescado: ciência, tecnologia, inovação e legislação, Atheneu. p.197-206. 
Ocvirk S, Wilson AS, Appolonia CN, Thomas TK, O'Keefe SJD (2019) Fiber, fat, and colorectal cancer: new insight into modifiable dietary risk factors. Current Gastroenterology Reports 21, article number 62. doi: 10.1007/s11894-019-0725-2

Oliveira MJM (2004) Estudo físico-químico e sensorial de produtos elaborados da carne triturada de acaráprata (Chaetobranchus semifasciatus). Relatório. Programa Institucional de Iniciação Científica: Universidade Federal do Amazonas, Manaus, 2004.

Ørnholt-Johansson G, Gudjónsdóttir M, Nielsen ME, Skytte JL, Frosch S (2017) Analysis of the production of salmon fillet - Prediction of production yield. Journal of Food Engineering 204: 80-87. doi: 10.1016/j.jfoodeng.2017.02.022

Raúl LJ, Araújo IB, Barbosa RC, Maciel MIS, Shinohara NKS, Oliveira Filho PRC (2018) Manufacture of biquara (Haemulon plumierii - Lacepède, 1801) fishburger with addition of wheat bran. Journal of Aquatic Food Product Technology 27(5): 544-556. doi: $10.1080 / 10498850.2018 .1461159$
Santos GM, Ferreira EJG, Zuanon, JAS (2006) Peixes Comerciais de Manaus, 2ed, Ibama/PróVarzea, p.144.

Silva SR, Fernandes ECS (2010) Aproveitamento da corvina (Argyrosomus regius) para elaboração do fishburger. Cadernos de Pesquisa 17(3).

Souza AFL, Inhamuns AJ (2011) Análise de rendimento cárneo das principais espécies de peixes comercializadas no Estado do Amazonas, Brasil. Acta Amazonica 4(2): 289-296.

Tacon AGJ, Lemos D, Metian M (2020) Fish for health: improved nutritional quality of cultured fish for human consumption. Reviews in Fisheries Science and Aquaculture. doi: 10.1080/23308249.2020.1762163

Viana AP, Inhamuns AJ, Oliveira PR, Souza LCL (2016) Efeito da embalagem com atmosfera modificada na conservação do Brycon amazonicus. Boletim do Instituto de Pesca 42(1): 17-28. doi: 10.5007/16782305.2016v42n1p17n1p17 